\title{
Thomson scattering from a shock front
}

\author{
A. B. Reighard, R. P. Drake, T. Donajkowski, M. Grosskopf, and K. K. Dannenberg \\ Department of Atmospheric, Oceanic, and Space Sciences, University of Michigan, \\ Ann Arbor, Michigan 48109 \\ D. Froula, S. Glenzer, J. S. Ross, and J. Edwards \\ Lawrence Livermore National Laboratory, Livermore, California 94551
}

(Received 8 May 2006; presented on 8 May 2006; accepted 29 May 2006; published online 22 September 2006)

\begin{abstract}
We have obtained a Thomson scattering spectrum in the collective regime by scattering a probe beam from a shock front, in an experiment conducted at the Omega laser at the Laboratory for Laser Energetics. The probe beam was created by frequency converting a beamline at Omega to a $2 \mathrm{~ns}$ pulse of $0.263 \mu \mathrm{m}$ light, focused with a dedicated optical focusing system. The diagnostic system included collecting optics, spectrometer, and streak camera, with a scattering angle of $101^{\circ}$. The target included a primary shock tube, a 20 - $\mu \mathrm{m}$-thick beryllium drive disk, $0.3-\mu \mathrm{m}$-thick polyimide windows mounted on a secondary tube, and a gas fill tube. Detected acoustic waves propagated parallel to the target axis. Ten laser beams irradiated the beryllium disk with $0.351 \mu \mathrm{m}$ light at 5 $\times 10^{14} \mathrm{~W} / \mathrm{cm}^{2}$ for $1 \mathrm{~ns}$ starting at $t_{o}$, driving a strong shock through argon gas at $\rho_{o}=1 \mathrm{mg} / \mathrm{cc}$. The $200 \mathrm{~J}$ probe beam fired at $t=19 \mathrm{~ns}$ for $2 \mathrm{~ns}$, and at $t=20.1 \mathrm{~ns}$ a $0.3 \mathrm{~ns}$ signal was detected. We attribute this signal to scattering from the shocked argon, before the density increased above critical due to radiative collapse. () 2006 American Institute of Physics. [DOI: 10.1063/1.2220069]
\end{abstract}

Thomson scattering measurements use an optical laser with some initial wavelength and wave number $\left(\lambda_{o}\right.$ and $\left.k_{o}\right)$ to elastically scatter from electron density fluctuations with a given wave vector $(\mathbf{k}){ }^{1,2}$ Over the last 40 years, experiments have used Thomson scattering to extract fundamental plasma properties from the scattered frequency spectrum, and it is now widely used as a diagnostic in fusion research. ${ }^{3-5}$

Thomson scattering probes individual electrons in the noncollective regime, when light is scattered from plasma fluctuations (of wave number $k$ ) with wavelengths shorter than a Debye length $\left(k \lambda_{\mathrm{De}}>1\right)$. Resonant plasma fluctuations in the collective regime, in the form of probed collective ion-acoustic wave features (of wave number $k_{\text {iaw }}$ ), are observed when the probed electrons follow the motion of the ions $\left(Z T_{e} / T_{i}>k_{\mathrm{iaw}} \lambda_{\mathrm{De}}\right.$, where $Z$ is ionization state, $T_{e}$ is electron temperature, and $T_{i}$ is ion temperature). In this regime the electrons move to screen the potential created by ion fluctuations. ${ }^{6}$ To probe electrons in the collective regime of a driven shock wave in gas, we have applied Thomsonscattering techniques to a radiatively collapsed shock wave in argon gas.

The experiment conducted for this purpose created a driven shock in argon with shock velocity high enough to cause radiative collapse. Thomson-scattered light was then collected from the shocked gas, and an attempt was made to image the dense, postshock gas using $\mathrm{x}$-ray radiography. To accomplish this, the targets were designed to form a laserdriven piston that drove the shock, and the target body was structured to create unobstructed paths for the probe entrance and scattered light exit paths. Beam timing was specified to allow for x-ray radiography of the shock, using an independently positioned backlighter target. The target for these experiments was based on those for driven radiative shock ex- periments described in Reighard et al., ${ }^{7,8}$ and fabricated at the University of Michigan. A three-dimensional (3D) computer-aided design (CAD) drawing of the experimental geometry in the target chamber is shown in Fig. 1.

To create a driven shock that may radiatively collapse, a low- $Z$, planar disk was launched by laser ablation pressure into a cylindrical volume filled with argon gas. This disk was fashioned from beryllium, $20 \pm 3 \mu \mathrm{m}$ thick, $2.0 \pm 0.1 \mathrm{~mm}$ in diameter. The disk was mounted on a polyimide tube, inside diameter (i.d.) $575 \mu \mathrm{m}$, outside diameter (o.d.) $600 \mu \mathrm{m}$. The target package was gas tight, and fitted at the back end with a hypodermic tube through which the target was evacuated of air and filled with argon. The argon pressure was measured for each experiment, and was $1.1( \pm 10 \%)$ atm for the cases of interest here, corresponding to $\rho_{o}=1 \mathrm{mg} / \mathrm{cm}^{3}$ or to $2.7 \times 10^{19}$ atoms $/ \mathrm{cm}^{3}$.

To allow for probe entrance and Thomson-scatteredlight-exit holes, another polyimide tube of larger diameter was fitted over the main target tube, creating an arm at a $45^{\circ}$ angle to the shock propagation axis, pointing towards the $4 \omega$ probe beam location when properly aligned. This polyimide tube was $725 \mu \mathrm{m}$ i.d., $875 \mu \mathrm{m}$ o.d., and totaled approximately $3 \mathrm{~mm}$ long. This arm served two main purposes. First, it acted as a guide to drill an entrance hole into the main target body, clearing a path to what would be the scattering volume on the shock axis. Second, it provided a flat surface onto which a thin film of polyimide could be affixed, sufficiently far from the target axis that plastic plasma would not mix with argon plasma on the shock axis, providing at least a $2 \mathrm{~mm}$ offset from the center of the main target tube. This thin film was $3000 \AA$ thick, and provided a gas tight barrier. Design calculations showed that the plastic plasma produced in the destruction of this film cleared from the 


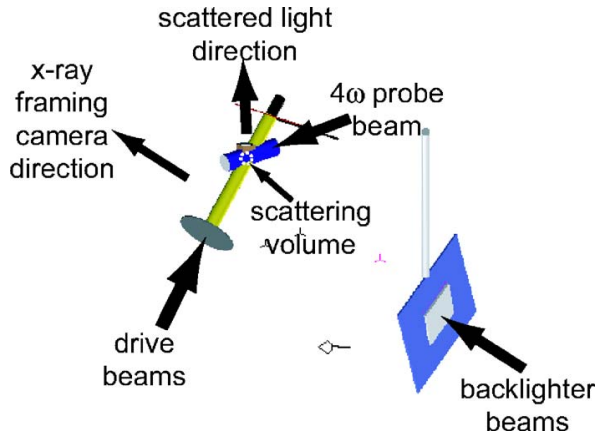

FIG. 1. 3D CAD rendering of experimental geometry, with beam directions. Beam timings and properties are discussed in the text. The gas fill tube was attached to plastic hose through a voltage transducer to measure target gas pressure. In addition, alignment wires used for metrology and rotational alignment were positioned at two separate places on the target. A dotted circle outlines the location of the scattering volume on the target axis.

probe beam path sufficiently quickly such that it did not interfere with the propagation of the probe beam. The alignment of this tube was checked during target metrology to ensure a clear path to the shock propagation axis, as this was sensitive to mounting angle and both rotational and translational alignment of the target in the chamber. Another hole was drilled in the main target body that faced the scattered light diagnostic when the target was properly positioned in the target chamber. This opening was approximately $400 \mu \mathrm{m}$ in diameter, drilled through a flat surface fashioned from epoxy, and was also covered with a 3000 - $\AA$-thick polyimide film. This provided an unobstructed path for scattered light towards the diagnostic when this film was destroyed in the experiment.

We focused ten laser beams of wavelength $0.35 \mu \mathrm{m}$ onto a $1 \mathrm{~mm}$ spot centered on the beryllium drive disk in a square, 1 ns flat-top pulse, with the midpoint of the rising edge defining time $t=0$. The total energy was $\leqslant 4000 \mathrm{~J}$. Distributed phase plates (DPPs) created super-Gaussian focal spots with a diameter of $820 \mu \mathrm{m}$ [full width at half maximum (FWHM)], with small-scale structure which fluctuated via smoothing by spectral dispersion (SSD). The resulting laser irradiance was up to $10^{15} \mathrm{~W} / \mathrm{cm}^{2}$. The pressure from laser ablation first shocked and then accelerated the drive disk, launching it into the argon and driving a shock in the argon gas. At $16.0 \pm 0.25 \mathrm{~ns}$ after the drive beams turned on, a single destroyer beam with an energy of $400 \mathrm{~J}$ and a spot size of $400 \mu \mathrm{m}$ fired on the film facing the UV spectrometer diagnostic in a 2 ns flat-topped pulse with no beam smoothing, clearing the path to this diagnostic. At $19.0 \pm 0.25 \mathrm{~ns}$ after the drive beams turned on, the probe beam turned on at $200 \mathrm{~J}$ in a $100 \mu \mathrm{m}$ spot with no beam smoothing, in a $2 \mathrm{~ns}$ flat-topped pulse, which first exploded the film on the arm facing this beam, as described above. This beam was aligned to target chamber center first using a frequency doubled beam, then confirmed in an alignment shot prior to the experiment. The alignment shot used the $4 \omega$ beam shooting a vanadium disk positioned at target chamber center. This shot also checked the successful propagation of the scattered light onto the UV spectrometer slit through target chamber center.

The probe beam came from port P9 $(\theta=116.57, \phi$ $=234.0)$ in the Omega chamber, and was a frequency quadrupled component of the main beam, at $\lambda=0.263 \mu \mathrm{m}$. The

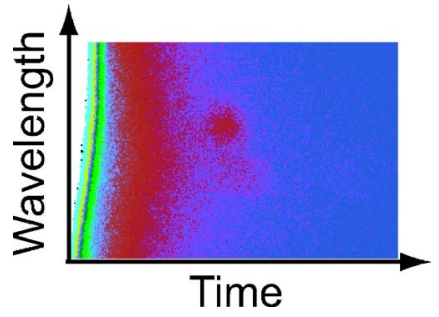

FIG. 2. Imaged spectrum from successful Thomson scattering experiment. Scattered light signal begins at $20.1 \pm 0.25 \mathrm{~ns}$ after drive beams turn on, about halfway through the 2 ns duration of the probe beam. The Thomson scattered light duration was $300 \pm 12$ ps. Timing and velocity measurements were consistent from scattering within the driven shock transition layer, as the gas collapses due to radiation loss.

probe-beam spot size was $100 \mu \mathrm{m}$, and with an energy of $200 \mathrm{~J}$. The collection diagnostic was an imaging UV spectrometer located in the TIM 2 (ten-inch manipulator) port $(\theta=79.19, \phi=90.0)$ of the Omega chamber, with a $250 \mu \mathrm{m}$ slit. In this geometry, the scattering angle was $101^{\circ}$. The target axis $(\theta=69.6, \phi=241.5)$ was chosen such that the ionacoustic waves probed via Thomson scattering were parallel to the shock propagation direction. The scattering volume in the target was $3.7 \pm 0.1 \mathrm{~mm}$ from the drive surface of the target. The volume was determined by the overlap of the $100 \mu \mathrm{m}$ spot size from the probe beam and the slit from the spectrometer.

The spectrometer used was an $f / 8.7,1 \mathrm{~m}$ imaging UV spectrometer with a grating with 3600 grooves $/ \mathrm{mm}$. The spectrometer dispersion was calibrated with lines from mercury lamp near $0.310 \mu \mathrm{m}$, with a standard adjustment to make the measurement appropriate for the $4 \omega$ probe beam wavelength of $0.263 \mu \mathrm{m}$. The central wavelength, $\lambda=0.263$, was confirmed by measuring stray $4 \omega$ light on an experiment in the same week. Spectra were imaged on a streak camera, with a wavelength dispersion of $0.036 \AA$ / pixel in a 5 ns window, with $6 \mathrm{ps} / \mathrm{pixel}$ dispersion in time.

In addition to Thomson scattering, we performed x-ray radiography of the target, using an x-ray framing camera opposite the backlighter target. Point projection radiography of the shock proved unsuccessful, as an $L$-shell backlighter proved too dim to effectively image the absorption features at that low an irradiance.

A double-peaked scattered-light spectrum was detected at $20.1 \pm 0.25 \mathrm{~ns}$ after the drive beams turned on, $1.1 \mathrm{~ns}$ after the probe beam turned on, about halfway through the duration of the probe beam. The signal lasted $300 \pm 12$ ps. The peaks were Doppler shifted by $+1.41 \pm 0.2 \AA$ by the plasma velocity of the gas in the scattering volume, corresponding to a plasma velocity of $110 \pm 8 \mathrm{~km} / \mathrm{sec}$. The timing of the signal, along with the measured plasma velocity, suggests the probe beam was scattered off of shocked plasma in the transition region of the radiatively shocked gas in argon, before the gas density increased above critical for this geometry. The imaged spectrum is shown in Fig. 2. Further data analysis is discussed in Reighard et al. ${ }^{9}$

The authors would like to thank the University of Michigan target fabrication team, including Mark Taylor, Christine Krauland, Donna Marion, and Douglas Kremer. The authors would also like to thank the technical staff at the Omega laser facility. This work is supported by the National Nuclear 
Security Agency under DOE Grant Nos. DE-FG0399DP00284 and DE-FG03-00SF22021, and by other grants and contracts.

${ }^{1}$ H.-J. Kunze, E. Fuenfer, B. Kronast, and W. H. Kegel, Phys. Lett. 11, 42 (1964).

${ }^{2}$ A. A. Offenberger, W. Blyth, E. Dangor, A. Djaoui, M. H. Key, Z. Naimudin, and J. S. Wark, Phys. Rev. Lett. 71, 3983 (1993).

${ }^{3}$ D. H. Froula, P. Davis, L. Divol, J. S. Ross, N. Meezan, D. Price, S. H.
Glenzer, and C. Rousseaux, Phys. Rev. Lett. 95, 195005 (2005).

${ }^{4}$ S. H. Glenzer et al., Phys. Rev. Lett. 82, 97 (1999).

${ }^{5}$ S. H. Glenzer et al., Phys. Rev. Lett. 79, 1277 (1997).

${ }^{6}$ J. Sheffield Plasma Scattering of Electromagnetic Radiation (Academic, New York, 1975).

${ }^{7}$ A. B. Reighard et al., Proceedings of the Third Inertial Fusion Science and Applications, edited by B. A. Hammel, D. D. Meyerhofer, J. Meyerter-Vehn, and H. Azechi (American Nuclear Society, Inc., 2004).

${ }^{8}$ A. B. Reighard et al., Phys. Plasmas (to be published).

${ }^{9}$ A. B. Reighard et al., Phys. Rev. Lett. (in preparation). 